\title{
Human amniotic membrane intra-articular injection prevents cartilage damage in an osteoarthritis model
}

\author{
I.A. MARINO-MARTÍNEZ ${ }^{1}$, A.G. MARTÍNEZ-CASTRO², V.M. PEÑA-MARTÍNEZ ${ }^{2}$, \\ C.A. ACOSTA-OLIVO ${ }^{2}$, F. VÍlCHEZ-CAVAZOS ${ }^{2}$, A. GUZMÁN-LÓPEZ ${ }^{3}$, EDELMIRO PÉREZ RODRÍGUEZ ${ }^{4}$, \\ V.J. ROMERO-DÍAZ ${ }^{5}$, J.A. ORTEGA-BLANCO ${ }^{6}$ and J. LARA-ARIAS ${ }^{2}$ \\ ${ }^{1}$ Pathology Service; ${ }^{2}$ Orthopedics and Traumatology Service; ${ }^{3}$ Obstetrics and Gynecology Service; \\ ${ }^{4}$ Transplants Service, Hospital Universitario 'Dr. José E. González'; ${ }^{5}$ Department of Histology, Facultad de Medicina, \\ Universidad Autónoma de Nuevo León, Monterrey, NL 64460; ${ }^{6}$ Top Health, SAPI de CV, Zapopan, JAL 45070, Mexico
}

Received March 8, 2017; Accepted August 9, 2018

DOI: $10.3892 /$ etm.2018.6924

\begin{abstract}
Osteoarthritis (OA) is a degenerative joint disease that affects the soft tissues and bones of involved articulations as a result of deregulation between synthesis and extracellular matrix degradation in articular cartilage. The present study evaluated the effect of intra-articular injection of human amniotic membrane (AM) as a treatment in an OA animal model in the knee. Chemical OA was developed in the knees of New Zealand rabbits. Once OA was established, the right knees only were treated with an intra-articular injection of human AM, with the left knees considered as a negative control group. The evaluation was performed at 3 and 6 weeks post-treatment. At 3 weeks post-injection, the cartilage exhibited fibrillation, erosion, cracks and cell clusters in the negative control group, but not in the treated group $(\mathrm{P}=0.028)$. At 6 weeks post-injection, the left knees exhibited hypertrophy, cracks, cell clusters, decreased matrix staining and structure loss. However, the right knees exhibited cell clusters without evidence of disruption in cartilage integrity $(\mathrm{P}=0.015)$. These results suggested that the intra-articular injection of human AM delays histological changes of cartilage in OA.
\end{abstract}

\section{Introduction}

Osteoarthritis (OA) is a multifactorial, degenerative and incapacitating disease that affects the soft tissues and bones of the involved articulations, causing pain and decreased function due to differences between the rate of synthesis and extracellular matrix degradation of articular cartilage (1-3).

Correspondence to: Dr J. Lara-Arias, Orthopedics and Traumatology Service, Hospital Universitario 'Dr. José E. González', Universidad Autónoma de Nuevo León, 104-C Av. Madero y Gonzalitos s/n, Monterrey, NL 64460, Mexico

E-mail: jorgelara77@gmail.com

Key words: osteoarthritis, articular cartilage, amniotic membrane
Nonsteroidal anti-inflammatory drugs and intra-articular steroid injections are commonly indicated as a first choice treatment in OA. Together with physical exercise and corporal weight control, the purpose of this treatment is to relieve pain and to improve articular function; however, it is not always effective. Although the intra-articular injection of hyaluronic acid has been approved as a knee OA treatment in patients for which non-drug treatment and simple analgesics have been unsuccessful, this treatment is palliative but not therapeutic (4).

The amniotic membrane (AM) is the innermost fetal membrane, usually discarded following birth as part of the placenta. The membrane itself and stem cells isolated from it have shown potential for applications in the regenerative medicine field, due to bacteriostatic and anti-angiogenic properties. It also has been shown that AM reduces pain, regulates the inflammatory process, and improves wound healing and epithelialization (5-8); it also exhibits low or no immunogenicity and acts as a physical barrier in the case of an exposed wound $(9,10)$. In clinical trials, complete AM has been evaluated for the treatment of skin burns (11), as a scaffold biomaterial in the reconstruction of the ocular surface (12), in head and neck surgery (13), and to prevent tissue adhesion in abdominal, head and pelvic surgery (14). Acellular AM has been evaluated in articular cartilage and tendon defects (15) and also in peripheral nerves (16).

Morphologically, AM is a thin and flexible membrane comprising a monolayer of epithelial cells aligned on a basal membrane, where the underlying stroma contains mesenchymal cells (17). These cells have the ability to differentiate into several lineages, including adipogenic, osteogenic, chondrogenic, hepatic, neurogenic and cardiomyogenic tissue (18-23). Amniotic cells are immunomodulatory in vitro (23), and the $\mathrm{AM}$ and allogeneic amniotic cells have been applied in clinical trials without evidence of immunologic rejection (10). The osteogenic capability of this membrane, and the fact it can act as a stimulator in repairing musculoskeletal injuries have been reported (24).

The aim of the present study was to analyze the histological changes produced by lyophilized and pulverized human AM, administered as a treatment in an OA model of the knee developed in rabbits. 


\section{Materials and methods}

Study type and ethics. The present study was an experimental, cross-sectional, comparative, prospective, simple blind study. The protocol was submitted to the Institutional Ethics and Research Committee of Universidad Autónoma de Nuevo León (Monterrey, Mexico) and approved (approval no. OR15-015).

AM isolation and processing. The placentas were obtained from healthy donors who were scheduled to have cesarean surgery in the obstetrics department of the Hospital General de Occidente (Zapopan, Mexico) from May 2017 to August 2017. The placentas were supplied by Top Health (Zapopan, Mexico) who provided proof of informed consent of the donors. Following separating of the placenta from the placental button, the chorionic membrane was then carefully separated. To decellularize the AM, it was washed with $15 \% \mathrm{NaCl}$ and gently mixed in $\mathrm{NaOH} 0.01 \mathrm{~N}$ until brown in color. $\mathrm{NaOH}$ was neutralized by adding ascorbic acid (1N). Hydrogen peroxide solution and gentle mixing were used to obtain a white color membrane, following which dehydration with $96 \%$ ethanol was performed. The membrane was placed to be dried for $24 \mathrm{~h}$ at room temperature in a ventilated area. The dried membrane was pulverized, and a screening was performed to determine the particle size. The membrane was lyophilized and irradiated with gamma rays of $25 \mathrm{kGy}$ prior to use in animals.

Chemical AO model. In the present study, six adult male New Zealand rabbits aged $\sim 3$ months old and with an average weight of $2.5 \mathrm{~kg}$ were obtained from the Animal Production Unit of the Facultad de Agronomia (Escobedi, Mexico). The experimental animals were anesthetized with an intramuscular injection of $1.9 \mathrm{mg} / \mathrm{kg}$ xylazine and $46 \mathrm{mg} / \mathrm{kg}$ ketamine. Intra-articular injections of $250 \mu 1$ of collagenase type II (Clostridium histolyticum type II, active enzyme $425 \mathrm{U} / \mathrm{mg}$, $4 \mathrm{mg} / \mathrm{ml}$; Thermo Fisher Scientific, Inc., Waltham, MA, USA) were performed at days 0 and 4 (25). The rabbits were housed in a bioterium for 3 and 6 weeks. Rabbits were kept in individual cages at a temperature of $21^{\circ} \mathrm{C}$ and a relative humidity of $55 \%$ with a $12 \mathrm{~h}$ light/dark cycle and ad libitum access to food and water.

Intra-articular infiltration of the AM. Once the OA model was established in both knees of all rabbits, the animals were divided into two observation groups, with three animals in each group. Each rabbit underwent an intra-articular infiltration in the right knee of the lyophilized human AM $(0.040 \mathrm{mg} / 0.200 \mathrm{ml})$ and saline solution $(0.6 \mathrm{ml})$ in the left knee (control group). The animals were sacrificed with an overdose of xylazine and ketamine IV at 3 and 6 weeks (Group 1 and 2, respectively). The knees were obtained from each rabbit and placed in formaldehyde solution for preservation. All previously identified samples were sent for histological examination.

Morphological and histological analysis. The macroscopic morphological analysis was performed using the scale published by Yoshioka et al (26) in which the grades are as follows: Grade 1, intact articular surface; grade 2, minimal fibrillation; grade 3 , evident fibrillation; grade 4, erosion with bone exposure (Table I).
Each knee sample was fixed and decalcified prior to being embedded in paraffin blocks. From these, $4-\mu \mathrm{m}$ sagittal sections were made and stained with hematoxylin and eosin (H\&E) for structure, cellularity and 'tidemark' integrity evaluation. Masson's trichrome staining was used for the extracellular matrix evaluation. Histopathological assessment of cartilage damage was performed following Mankin's scale (27) (Table I). This scale assigns a histological score by adding the corresponding values to the changes in the cartilage structure, cellularity, matrix staining and in the 'tidemark' or basophilic limiting line. In this way, normal cartilage corresponds to 0 points and the most severe cartilage affectation corresponds to 14 points. The morphologic and histopathologic evaluations were performed by independent observers who did not know which group they assessed. Slides were observed using a Nikon fluorescent microscope (E600; Nikon Corporation, Tokyo, Japan).

Statistical analysis. The sample size calculation for animal models with $\alpha=0.05, \beta=0.08$ (one-tailed) was six individuals per group. Calculating a sample size for comparison of related averages $\alpha=0.05, \beta=0.08$ (two-tailed), taking a minimum difference of 2.5 , it was seven individuals per group. The numerical variables were analyzed using one-way analysis of variance with Tukey's multiple comparisons post-hoc test to estimate the differences between groups.

Significance was determined at $\mathrm{P} \leq 0.05$ was considered to indicate a statistically significant difference. Descriptive and inferential statistical analyses were performed using STATA-10-08 software (Stata Corporation, College Station, TX, USA).

\section{Results}

Human AM prevents macroscopic changes in the cartilage of $O A$ model. In the left knees of group 1, cartilage fibrillation and color changes were observed on the joint surface compared with the right knees which showed a macroscopic apparently intact surface. However, small areas with poor fibrillation were observed in the right knees (Fig. 1A and B). Despite these data, a statistically significant difference $(\mathrm{P}=0.024)$ was found when Yoshioka's scale scores of the left knees $(3.15 \pm 0.73)$ and right knees $(2.36 \pm 0.76)$ were compared.

In the left knees of group 2, subchondral bone was observed in regions of the femoral condyles and the tibial plateau (Fig. 1C and D). The left knees were assigned a score of $4.25 \pm 0.32$ according to Yoshioka's scale evaluation, whereas the right knees were assigned a score of $1.29 \pm 0.49(\mathrm{P}=0.015$; Table I).

Human AM protects from extracellular matrix destruction in the OA model. On examining the cartilage morphology in left knees of the group 1, increased fibers in the superficial cartilage zone and irregularities on the surface were observed. Compared with the right knees of the same group, the cartilage surface was more complete and continuous, with less fibrillation or observed indentations (Fig. 2A and B).

When the cellularity and matrix staining were evaluated, the left knees exhibited more cell clusters and reduced staining in regions where joint surface fibrillation was detected. By contrast, the right knees exhibited fewer and isolated cell clusters, with no decrease or loss of staining density. Overall, a 
Table I. Yoshioka's scale for macroscopic evaluation and Mankin's scale for histological evaluation.

\begin{tabular}{lccc}
\hline Group & Time post-injection (weeks) & Left knee injury & Right knee injury+AM \\
\hline Yoshioka's scale & 3 & & 0.024 \\
1 & 6 & $3.15 \pm 0.73$ & 0.015 \\
2 & & $4.25 \pm 0.32$ & $1.29 \pm 0.49$ \\
Mankin's scale & 3 & $4.33 \pm 0.67$ & $2.44 \pm 0.21$ \\
1 & 6 & $6.54 \pm 0.43$ & $1.87 \pm 0.73$ \\
2 & & & 0.028 \\
\hline
\end{tabular}

AM, amniotic membrane.
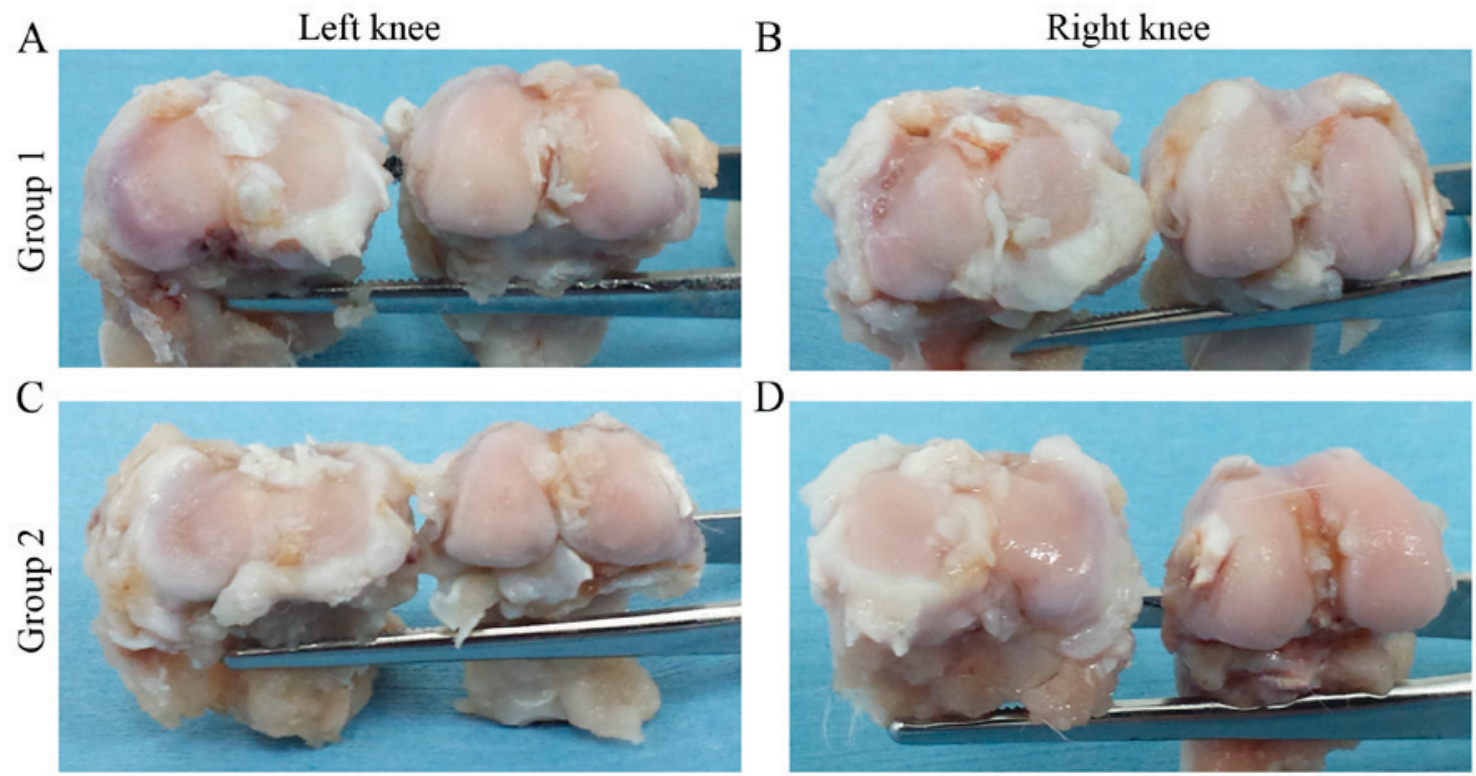

Figure 1. (A-D) Macroscopic analysis of representative knee joints. OA disease progression in the control group at (A) 3 and (C) 6 weeks. AM treatment group at (B) 3 and (D) 6 weeks post-AM infiltration. OA, osteoarthritis; AM, amniotic membrane.

significant statistical difference was observed when comparing the left and right knees of group $1(\mathrm{P}=0.028)$.

In group 2, the $\mathrm{H} \& \mathrm{E}$ (Fig. 2C and D) and Masson' trichrome staining (Fig. 3A and B) showed cracks, loss of structure, recesses, erosion, and fibrillation in the left knee cartilage. Abundant cell clusters and decreased staining were observed on the surface and extracellular matrix. The right knees of group 2 exhibited an almost intact surface with minimal articular fibrillation, no cracks and no evident surface erosion (Figs. 2 and 3). A number of cell clusters were observed in isolated areas where the cartilage surface was not compromised. Normal staining was observed on the cartilage surface and extracellular matrix.

For groups 1 and 2, there was no evident 'Tidemark' injury and statistical differences were found on comparing the right and left knees for Mankin's evaluation scale (Table I; $\mathrm{P}=0.028$ and $\mathrm{P}=0.015$ ).

\section{Discussion}

The AM has been investigated mainly for ophthalmologic applications, including regenerative medicine in the cornea (12). A number of studies investigating AM use in articular cartilage lesion regeneration have been reported and a chondroprotective effect with reduced proteoglycan loss, preventing damage progression of the extracellular matrix, were demonstrated (28). In a study investigating the injection of dehydrated human amnio/chorionic product, fewer defects and smaller lesion volumes were observed when compared with saline solution-injected control animals at day 21 post-injection; the study demonstrated cartilage destruction attenuation with increases in cartilage thickness and volume, and a decrease in total lesion area in animals injected with particulate AM (29). However, this previous study used a model in Lewis rats that underwent medial meniscal transection surgery to induce OA and did not perform histopathological analysis, which is considered to be the 'gold standard' for evaluating the potential therapeutics for OA. In the present study, a chemical OA model in rabbits was used and, as the joints of these animals are larger than the knees of rats, this may provide further support to the significance to the results.

In another study, in which a particulate AM and umbilical cord tissue in an OA model were used, their results were compared with EPIC-micro CT and histopathology to demonstrate a significant reduction in cartilage degeneration and 

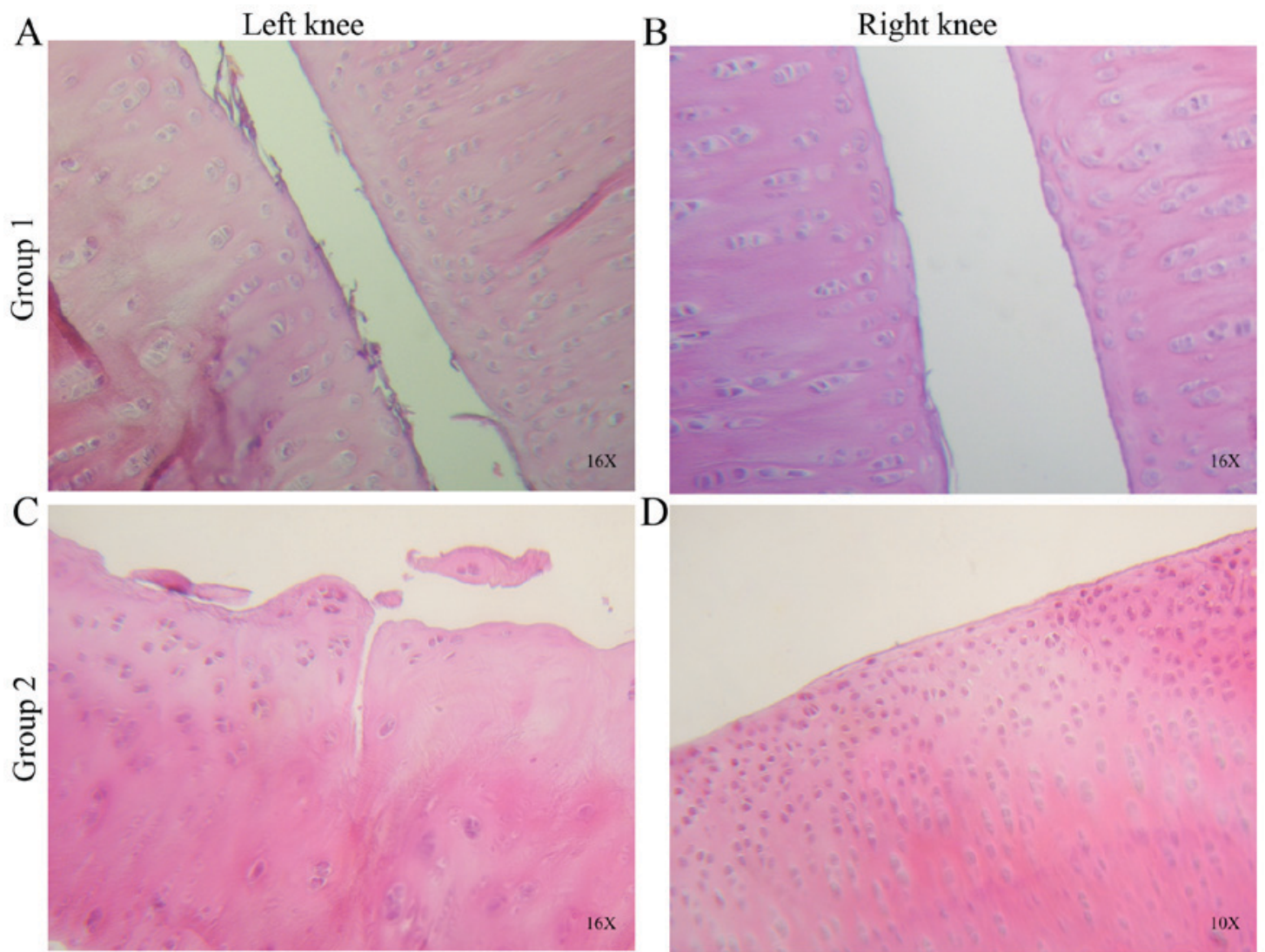

Figure 2. Histological sections of control and treated knees stained with hematoxylin and eosin. (A) Joint surface irregularities, fibrillation and formation of cell clones or 'clusters' were observed at 3 weeks in the control. (B) Joint surfaces with minimal fibrillation, but cellular clusters remained present in the treated knee. (C) Cracks were observed in the intermediate cartilage zone, with irregularities, fibrillation and cell clusters on the joint surface at 6 weeks in the control. (D) Hypercellularity, cell clusters and minimal fibrillation were observed in the articular cartilage surface of the treated knee. Images are at x10 and $\mathrm{x} 16$ objectives.
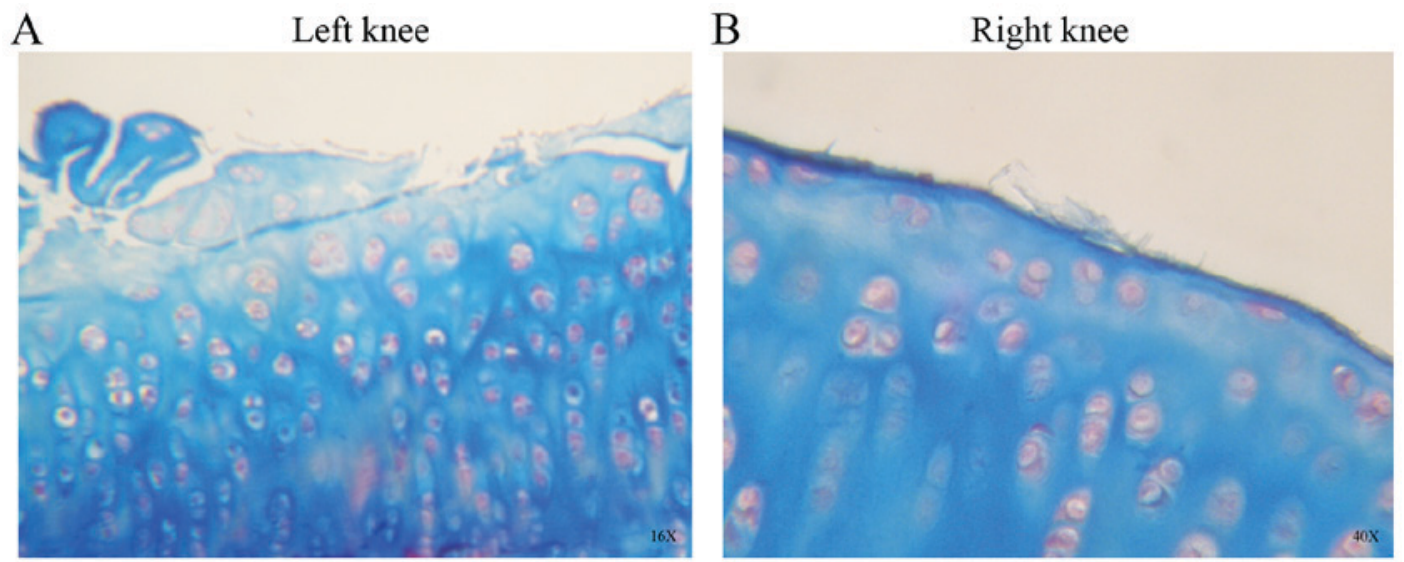

Figure 3. Histological sections of group 2 stained with Masson's trichrome staining. (A) Articular surface was eroded and cracked, with cell clusters and hypertrophic areas in the OA control knees. (B) Following amniotic membrane treatment, articular cartilage showed some irregularities on the surface, with fibrillation, cell clusters and hypercellular regions. Images are at x16 and x40 objective.

calcified cartilage at week 1 post-injection, providing further evidence that $\mathrm{AM}$ assists in preventing and treating OA (30).

he present study demonstrated that pulverized AM injected intra-articularly improved histological features of OA cartilage in rabbit knees, decreased disease progression and delayed histological changes, including loss of extracellular matrix staining. This is possibly due to it being responsible for supplying growth factors, that allow adequate re-epithelialization and epithelial cell migration. The low or absent immunogenicity of AM represents an advantage that reduces complications. However, it remains necessary to obtain further scientific evidence prior to indicating that AM is safe for its use in humans as a treatment for OA of the knee. Therefore, a novel animal model of a higher level in the phylogenetic scale is required to investigate the efficacy and safety of the AM application. 
Although the majority of the studies involving AM use animal models to reproduce OA disease, a number of studies have used AM for clinical trials in human volunteers. Vines et al (31) demonstrated the intraarticular injection feasibility of cryogenically preserved human amniotic suspension allografts for human patients suffering OA of the knee. Díaz-Prado et al (32) used human AM as a scaffold in human articular cartilage repair, and demonstrated that cryopreserved human AM can be used to support chondrocyte proliferation (32). Previous studies in tissue engineering have used cultured chondrocytes on the AM for use as a scaffold, and the results indicated the presence of collagen type II and an extracellular matrix similar to hyaline cartilage $(32,33)$. This fact may be due to the AM having a high content of transforming growth factor- $\beta 1$, and this protein upregulates chondrogenic gene expression $(34,35)$.

These results of the present study suggested that the treatment of early $\mathrm{OA}$ is feasible using an AM with different presentations, either fresh or cryopreserved. The present study used lyophilized, pulverized AM in saline solution as a vehicle for resuspension. This presentation is easy to handle and store, and reduces the infection risk as it is dried until resuspension in PRP. However, further investigations are required to determine the safety and efficacy of AM in clinical trials for the treatment of OA and other orthopedic problems, including ligament sprains, and fracture complications, including pseudoarthrosis, among others.

\section{Acknowledgements}

The present study was supported by The Orthopedics and Traumatology Service, Hospital Universitario 'Dr. José E. González', Universidad Autónoma de Nuevo León, and Top Health SAPI de CV.

\section{Funding}

Not applicable.

\section{Availability of data and materials}

The datasets used and/or analyzed during the present study are available from the corresponding author on reasonable request.

\section{Authors' contributions}

IAMM analyzed and interpreted the data. AGMC established the animal model. VJRD performed the histological knee examination. VMPM participated in the design of the protocol for animal model development. CAAO examined the histological sections as a blind observer and contributed to the statistical analysis. FVC participated in the interpretation of results and the writing of the final manuscript. FVC was also responsible for processing amniotic membranes at the Bone and Tissue Bank of the Universidad Autónoma de Nuevo León prior to use in the study. AGL obtained knees following euthanasia and processed sections using histological techniques. EPR contributed to the experiments. JAOB participated in the construction of the Discussion section of the final manuscript and reviewing the English. JLA contributed to the design of the study, the reviewing of all procedures and the writing of the final manuscript. All authors read and approved the final manuscript.

\section{Ethics approval and consent to participate}

The protocol was submitted to the Institutional Ethics and Research Committee of Universidad Autónoma de Nuevo León and approved (approval no. OR15-015). Informed consent was provided by the donors of placentas.

\section{Patient consent for publication}

Not applicable.

\section{Competing interests}

The authors confirm that they have no competing interests.

\section{References}

1. Felson DT, Lawrence RC, Dieppe PA, Hirsch R, Helmick CG, Jordan JM, Kington RS, Lane NE, Nevitt MC, Zhang Y, et al: Osteoarthritis: New insights. Part 1: The disease and its risk factors. Ann Intern Med 133: 635-646, 2000.

2. Giménez Basallote S, Pulido Morillo FJ, and Trigueros Carrero JA (eds): Definición, etiopatogenia, factores de riesgo y pronóstico. In: Guía de Buena Práctica Clínica en Artrosis. 2nd edition. International Marketing \& Communication, SA (IM\&C), Madrid, Spain, pp11-17, 2008 (In Spanish).

3. Ajadi RA, Otesile EB and Kasali OB: Short-term changes in lipid profile following experimental osteoarthritis in dogs. Bulgarian J Veterinary Med 15: 166-171, 2012.

4. Anitua E, Sanchez M, Nurden AT, Zalduendo MM, de la Fuente M, Azofra J and Andía I: Platelet-released growth factors enhance the secretion of hyaluronic acid and induce hepatocyte growth factor production by synovial fibroblasts from arthritic patients. Rheumatology (Oxford) 46: 1769-1772, 2007.

5. Gajiwala K and Gajiwala AL: Evaluation of lyophilized, gamma-irradiated amnion as a biological dressing. Cell Tissue Bank 5: 73-80, 2004.

6. Gruss JS and Jirsch DW: Human amniotic membrane: A versatile wound dressing. Can Med Assoc J 118: 1237-1246, 1978.

7. Subrahmanyam M: Amniotic membrane as a cover for microskin grafts. Br J Plast Surg 48: 477-478, 1995.

8. Ward DJ, Bennett JP, Burgos H and Fabre J: The healing of chronic venous leg ulcers with prepared human amnion. Br J Plast Surg 42: 463-467, 1989.

9. Adinolfi M, Akle CA, McColl I, Fensom AH, Tansley L, Connolly P, Hsi BL, Faulk WP, Travers P and Bodmer WF: Expression of HLA antigens, beta 2-microglobulin and enzymes by human amniotic epithelial cells. Nature 295: 325-327, 1982.

10. Akle CA, Adinolfi M, Welsh KI, Leibowitz S and McColl I: Immunogenicity of human amniotic epithelial cells after transplantation into volunteers. Lancet 2: 1003-1005, 1981.

11. Faulk WP, Matthews R, Stevens PJ, Bennett JP, Burgos H and His BL: Human amnion as an adjunct in wound healing. Lancet 1: 1156-1158, 1980.

12. Kim JC and Tseng SC: Transplantation of preserved human amniotic membrane for surface reconstruction in severely damaged rabbit corneas. Cornea 14: 473-484, 1995.

13. Zohar Y, Talmi YP, Finkelstein Y, Shvili Y, Sadov R and Laurian N: Use of human amniotic membrane in otolaryngologic practice. Laryngoscope 97: 978-980, 1987.

14. Rennekampff HO, Dohrmann P, Föry R and Fändrich F: Evaluation of amniotic membrane as adhesion prophylaxis in a novel surgical gastroschisis model. J Invest Surg 7: 187-193, 1994.

15. He Q, Li Q, Chen B and Wang Z: Repair of flexor tendon defects of rabbit with tissue engineering method. Chin J Traumatol 5: 200-208, 2002. 
16. Mligiliche N, Endo K, Okamoto K, Fujimoto E and Ide C: Extracellular matrix of human amnion manufactured into tubes as conduits for peripheral nerve regeneration. J Biomed Mater Res 63: 591-600, 2002.

17. Parolini O, Alviano F, Bagnara GP, Bilic G, Buhring HJ, Evangelista M, Hennerbichler S, Liu B, Magatti M, Mao N, et al: Concise review: Isolation and characterization of cells from human term placenta: Outcome of the firstimtermationadurondxkshop on placenta derived stem cells. Stem Cells 26: 300-311, 2008.

18. Miki T, Lehmann T, Cai H, Stolz DB and Strom SC: Stem cell characteristics of amniotic epithelial cells. Stem Cells 23: 1549-1559, 2005.

19. Portmann Lanz CB, Schoeberlein A, Huber A, Sager R, Malek A, Holzgreve W and Surbek DV: Placental mesenchymal stem cells as potential autologous graft for pre- and perinatal neuro regeneration. Am J Obstet Gynecol 194: 664-673, 2006.

20. Sakuragawa N, Kakinuma K, Kikuchi A, Okano H, Uchida S, Kamo I, Kobayashi M and Yokoyama Y: Human amnion mesenchyme cells express phenotypes of neuroglial progenitor cells. J Neurosci Res 78: 208-214, 2004.

21. Wolbank S, Peterbauer A, Fahrner M, Hennerbichler S, van Griensven M, Stadler G, Redl H and GabrielC: Dose-dependent immunomodulatory effect of human stem cells from amniotic membrane: A comparison with human mesenchymal stem cells from adipose tissue. Tissue Eng 13: 1173-1183, 2007.

22. Zhao P, Ise H, Hongo M, Ota M, Konishi I and Nikaido T: Human amniotic mesenchymal cells have some characteristics of cardiomyocytes. Transplantation 79: 528-535, 2005.

23. Stadler G, Hennerbichler S, Lindenmair A, Peterbauer A, Hofer K and van Griensven M, Gabriel C, Redl H and Wolbank S: Phenotypic shift of human amniotic epithelial cells in culture is associated with reduced osteogenic differentiation in vitro. Cytotherapy 10: 743-752, 2008.

24. Lindenmair A, Wolbank S, Stadler G, Meinl A, Peterbauer-Scherb A, Eibl J, Polin H, Gabriel C, van Griensven M and Redl H: Osteogenic differentiation of intact human amniotic membrane. Biomaterials 31: 8659-8665, 2010.

25. Kikuchi T, Sakuta T and Yamaguchi T: Intra-articular injection of collagenase induces experimental osteoarthritis in mature rabbits. Osteoarthritis Cartilage 6: 177-186, 1998.

26. Yoshioka M, Coutts RD, Amiel D and Hacker SA: Characterization of a model of osteoarthritis in the rabbit knee. Osteoarthritis Cartilage 4: 87-98, 1996.
27. Mankin HJ, Dorfman H, Lippiello L and Zarins A: Biochemical and metabolic abnormalities in articular cartilage from osteo-arthritic human hips. II. Correlation of morphology with biochemical and metabolic data. J Bone Joint Surg Am 53: 523-537, 1971.

28. Buckland J: Osteoarthritis: Blocking cartilage damage in a rat model of OA by intra-articular injection of an amniotic membrane allograft. Nat Rev Rheumatol 10: 198, 2014.

29. Willett NJ, Thote T, Lin AS, Moran S, Raji Y,Sridaran S, Stevens HY and Guldberg RE: Intraarticular injection of micronized dehydrated human amnion/chorion membrane attenuates osteoarthritis development. Arthritis Res Ther 16: R47, 2014

30. Raines AL, Shih MS, Chua L, Su CW, Tseng SC and O'Connell J: Efficacy of particulate amniotic membrane and umbilical cord tissues in attenuating cartilage destruction in an osteoarthritis model. Tissue Eng Part A 23: 12-19, 2017.

31. Vines JB, Aliprantis AO, Gomoll AH and Farr J: Cryopreserved amniotic suspension for the treatment of knee osteoarthritis. J Knee Surg 29: 443-450, 2016.

32. Díaz-Prado S, Rendal-Vázquez ME, Muiños-López E, Hermida-Gómez T, Rodríguez-Cabarcos M, Fuentes-Boquete I, de Toro FJ and Blanco FJ: Potential use of the human amniotic membrane as a scaffold in human articular cartilage repair. Cell Tissue Bank 11: 183-195, 2010.

33. Lucero JM, Castiglioni AE, Hovanyecz P, Gorla A, Berasategui O, Fedrigo GV and Lorenti AS: Culture of chondrocytes on an acellular matrix derived from amniochorionic membrane. Rev Asoc Argent Ortop Traumatol 77: 207-212, 2012. (In Spanish)

34. Bischoff M, Stachon T, Seitz B, Huber M, Zawada M, Langenbucher A and Szentmáry N: Growth factor and interleukin concentrations in amniotic membrane-conditioned medium. Curr Eye Res 42: 174-180, 2017.

35. Kim YI, Ryu JS, Yeo JE, Choi YJ, Kim YS, Ko K and Koh YG: Overexpression of TGF- $\beta 1$ enhances chondrogenic differentiation and proliferation of human synovium-derived stem cells. Biochem Biophys Res Commun 450: 1593-1599, 2014.

This work is licensed under a Creative Commons Attribution-NonCommercial-NoDerivatives 4.0 International (CC BY-NC-ND 4.0) License. 